\title{
THE PRESENT AND FUTURE OF JUDGEMENT AGGREGATION THEORY. A LAW AND ECONOMICS PERSPECTIVE ${ }^{1}$
}

\author{
by Philippe Mongin \\ CNRS \& HEC Paris ${ }^{2}$
}

July 2018

\author{
Forthcoming in The Future of Economic Design, \\ edited by Jean-François Laslier, Hervé Moulin, Remzi Sanver and \\ William S. Zwicker, \\ to be published by Springer
}

\begin{abstract}
This chapter briefly reviews the present state of judgment aggregation theory and tentatively suggests a future direction for that theory. In the review, we start by emphasizing the difference between the doctrinal paradox and the discursive dilemma, two idealized examples which classically serve to motivate the theory, and then proceed to reconstruct it as a brand of logical theory, unlike in some other interpretations, using a single impossibility theorem as a key to its technical development. In the prospective part, having mentioned existing applications to social choice theory and computer science, which we do not discuss here, we consider a potential application to law and economics. This would be based on a deeper exploration of the doctrinal paradox and its relevance to the functioning of collegiate courts. On this topic, legal theorists have provided empirical observations and theoretical hints that judgment aggregation theorists would be in a position to clarify and further elaborate. As a general message, the chapter means to suggest that the future of judgment aggregation theory lies with its applications rather than its internal theoretical development.

Keywords: judgment aggregation theory, logical aggregation theory, law and economics, doctrinal paradox, discursive dilemma, canonical theorem of judgment aggregation theory, premiss-based versus conclusion-based method, collegiate courts, issue-based versus case-based adjudication method.
\end{abstract}

JEL Classification: D70, D71, D79, K40, K41

\footnotetext{
${ }^{1}$ The author gratefully acknowledges comments by Franz Dietrich, Samuel Ferey, Umberto Grandi and Jean-François Laslier.

${ }^{2} 1$ rue de la Libération, F-78350 Jouy-en-Josas. Email address: mongin@greg-hec.com
} 


\section{A new brand of aggregation theory}

It is a commonplace idea that collegial institutions generally make better decisions than those in which a single individual is in charge. This optimistic view, which can be traced back to French Enlighment social theorists like Rousseau and Condorcet, permeates today's western judiciary organization, which normally entrusts collegial courts with the competence to rule on the more complex cases; think of constitutional courts like the U.S. Supreme Court with its nine judges. However, the following, by now classic example from legal theory challenges the orthodoxy. A plaintiff has brought a civil suit against a defendant, alleging a breach of contract between them. The court is composed of three judges $A, B$ and $C$, who will determine whether or not the defendant must pay damages to the plaintiff $(d$ or $\neg d$ ). The case brings up two issues, i.e., whether the contract was valid or not ( $v$ or $\neg v$ ), and whether the defendant was or was not in breach of it $(b$ and $\neg b)$. Contract law stipulates that the defendant must pay damages if, and only if, the contract was valid and the defendant was in breach of it. Suppose that the judges have the following views of the two issues and accordingly of the case:

\begin{tabular}{|l|l|l|l|}
\hline$A$ & $v$ & $\neg b$ & $\neg d$ \\
\hline$B$ & $\neg v$ & $b$ & $\neg d$ \\
\hline$C$ & $v$ & $b$ & $d$ \\
\hline
\end{tabular}

In order to rule on the case, the court can either directly collect the judges' recommendations on it, or collect the judges' views of the issues and then solve the case by applying contract law to these data. If the court uses majority voting, the former, case-based method delivers $\neg d$, whereas the latter, issuebased method returns first $v$ and $b$, and then $d$. This elegant example is due to legal theorists Kornhauser and Sager (1993, with a preliminary sketch in 1986). They describe as a doctrinal paradox any similar occurrence in which the two methods give conflicting answers. What makes the discrepancy paradoxical is that each method is commendable on some ground, i.e., the former respects the judges' final views, while the latter provides the court with a rationale, so one would wish them always to be compatible. The legal literature has not come up with a clear-cut solution, although the issue-based method often attracts more sympathy. This persisting difficulty casts doubt on the belief that collegial courts would be wiser than individual ones. Clearly, with a single judge, the two methods coincide unproblematically.

An entire body of work, now referred to as judgment aggregation theory, has grown out of Kornhauser and Sager's doctrinal paradox. As an intermediate step, their problem was rephrased by political philosopher Pettit (2001), who wanted to make it both more widely applicable and more analytically tractable. What he calls the discursive dilemma is, first of all, the generalized version of 
the doctrinal paradox in which a group, whatever it is, can base its decision on either the conclusion-based or the premiss-based method, whatever the substance of conclusions and premisses. Any time the propositions of interest to the group can logically be divided into a set of premisses and a conclusion, the representative of the group can put to the vote either the conclusion directly or the premisses themselves, while performing the required inference in the latter case. What the court example illustrates can apply equally well to a political assembly, an expert committee, and many deliberating groups; as one of the promoters of the concept of deliberative democracy, Pettit would speculatively add political society as a whole. Second, and more technically, the discursive dilemma shiftes the stress away from the conflict of two methods of decision to the logical contradiction within the total set of propositions that the group accepts. In the previous example, with $d \longleftrightarrow v \wedge b$ representing contract law, the contradictory set is

$$
\{v, b, d \longleftrightarrow v \wedge b, \neg d\} .
$$

This shift has far-reaching consequences since all propositions are now being treated alike; indeed, the very distinction between premisses and conclusion vanishes. If one is concerned with developing a general theory, this move has clear advantages. Given a set of propositions, it may be tricky to divide it appropriately, and thus to say what the two methods exactly are. It is definitely simpler, and it may be sufficient, to pay attention to whole sets of propositions accepted by either the individuals or the group, more briefly judgment sets, and inquire how these relate to each other. The question raised by the discursive dilemma is why and when individual judgment sets that are consistent deliver a collective judgment set that is inconsistent. This is already the problem of judgment aggregation.

In a further step, List and Pettit (2002) introduced an aggregation mapping $F$, which takes profiles of individual judgment sets $\left(A_{1}, \ldots, A_{n}\right)$ to collective judgment sets $A$, and subject $F$ to formal conditions which they demonstrate are logically incompatible. Both the proposed formalism and impossibility conclusion are in the vein of social choice theory, but they are directed at the discursive dilemma, which the latter theory cannot handle in its own terms, since there is no question here of preferences and related social-choice-theoretic concepts. At this stage, judgment aggregation theory exists in full, having defined its object of study - the $F$ mapping, or collective judgment function - as well as its method of analysis - it consists in defining axiomatic conditions on $F$ and investigating when these conditions result into impossibilities, or to the contrary, support well-behaved rules, such as simple majority voting, the only rule considered in the above example.

List and Pettit's impossibility theorem was shortly superseded by others of growing sophistication, due to Pauly and van Hees (2006), Dietrich (2006), Dietrich and List (2007a), Mongin (2008), Nehring and Puppe (2008, 2010), Dokow and Holzman (2009, 2010a and b), Dietrich and Mongin (2010a). This 
lengthy, but still incomplete list, should be complemented by two papers that contributed differently to the progress of the field. Renewing the investigation of social choice rules, Nehring and Puppe (2002) characterized, in the sense of necessary and sufficient conditions, those preference domains for which relevant sets of axioms - strategy-proofness among them - entail dictatorship. Judgment aggregation theory has taken up this concern for characterizing impossibility domains. It does so by defining the agenda of propositions on which the individuals and the collective make judgments, and then by asking which properties of the agenda turn any $F$ satisfying certain axioms into a dictatorship (or some other unpalatable rules like oligarchy). On a very different score, Dietrich (2007) showed that the logical formalism of judgment aggregation theory could be deployed without making reference to any specific logical calculus. Only a few elementary properties of the formal language and of the logic need assuming for the theorems to carry through. The so-called general logic states these requisites (see Dietrich and Mongin, 2010a, for an up-to-date version). The first papers were unnecessarily restrictive in relying on the elementary propositional calculus alone.

Implicit in the previous comments is the view that judgment aggregation theory is a brand of logical theory. This view fits the work of many judgment aggregation theorists very well, but not so well the work of Nehring and Puppe (whose contributions often use the framework of social choice theory) or the work of Dokow and Holzman (who have devised a combinatorial framework of their own, drawing inspiration from Rubinstein and Fishburn, 1986). The more thorough review by Mongin (2012) elaborates on this difference in perspectives while defending the use of logic (for an earlier French version, see Dietrich and Mongin, 2010b). This review also points out the historical origin of judgment aggregation theory, arguably already understood as a logical theory, in Guilbaud (1952) and Condorcet himself, whose rediscovery in the mid-20th century was largely due to Guilbaud.

Consistently with this orientation towards logic, section 2 introduces a syntactical framework for the $F$ function and states in this framework the axiomatic conditions on $F$ that have attracted most attention. The crucial issue of agendas arises in section 3, which characterizes those which, for given conditions on $F$, turn this function into a dictatorship (oligarchy will not be discussed here). Essentially, there is a single theorem, but in three forms, each of which is instructive in itself. This theorem is the central achievement of judgment aggregation theory by common consent, so one can take it to represent, at least in very broad outlook, the present of that theory. Section 4 tentatively argues about its future, a challenging and unavoidably questionable exercise. It seems as if the years of high theorizing are over by now, and in the recent years, judgment aggregation theory has indeed moved forward mostly on the front of applications. In our view, this applied work delineates the near future of the theory, and we accordingly restrict attention to it in the prospective part of this chapter. 
The first applications of judgment aggregation theory were to social choice theory. Having been a heuristic source for the framework and conditions on $F$, this field was also a convenient source of corollaries to the main theorems. It is sufficient to replace the abstract propositional symbols $p, q, r \ldots$ by formulas such as $x R y, z P w, u I v$, with the usual preference interpretations, to give rise to a tentative application. Whether a proper social choice corollary follows will depend in particular on whether the agenda conditions stated in the judgment aggregation theorems hold for the new set of formulas, given the logical connections between these formulas (reflecting transitivity, completeness, acyclicity, and the like). Arrow's theorem can be recovered in this way, along with variants that either already existed or are new to social choice theory. The second and actually larger group of applications has emerged at the crossroads of formal epistemology and theoretical computer science. Among others, it is concerned with relaxing those judgment aggregation conditions which are responsible for dictatorship. This has led to the flourishing "belief merging literature", which often adopts a syntactical form and often pays attention to computational issues, as befit its computer science connections. For this fairly large body of work, we refer to Grossi and Pigozzi (2014) and Pigozzi (2016). The present, purposefully short discussion deals with a third group of applications, concerning law and economics, which have not yet been explored. Given that judgment aggregation theory originates in a law and economics example, it is but natural to inquire what it can contribute to this field in return. Section 4 is entirely devoted to these applications, which will be seen to be mostly prospective, rather than already existing.

\section{A logical framework for judgment aggregation theory}

By definition, a language $\mathcal{L}$ for judgment aggregation theory is any set of formulas $\varphi, \psi, \chi, \ldots$ that is constructed from a set of logical symbols $\mathcal{S}$ containing $\neg$, the Boolean negation symbol, and that is closed for this symbol (i.e., if $\varphi \in \mathcal{L}$, then $\neg \varphi \in \mathcal{L})$. Normally, $\mathcal{S}$ contains symbols for other Boolean connectives (conjunction $\wedge$, disjunction $\vee$, implication $\rightarrow$, biimplication $\longleftrightarrow$ ) and in principle, it may contain modal operators (such as $\square$ interpreted as "it is obligatory that", $B_{i}$ interpreted as "individual $i$ believes that", or $\Longrightarrow$ interpreted as a non-Boolean conditional). Then, these added symbols will have to satisfy the relevant closure properties. A logic for judgment aggregation theory is any set of axioms and rules that regulates the inference relation $\vdash$ on $\mathcal{L}$ and associated technical notions - logical truth and contradiction, consistent and inconsistent sets - while satisfying the general logic. Informally, the latter stipulates that $\vdash$ be monotonic and compact, and that any consistent set of formulas can be extended to a complete consistent set $(S \subset \mathcal{L}$ is complete if, for all $\varphi \in \mathcal{L}$, either $\varphi \in S$ or $\neg \varphi \in S$ ). Monotonicity means that inductive logics are excluded, and compactness (which is needed only in specific proofs) that some deductive logics 
are. The extendability requisite is standard for deductive logics.

Among the many calculi that enter this framework, propositional examples stand out, but they can be richer than the elementary propositional calculus with Boolean connectors alone. Adding modal operators, one gets the many calculi of deontic, epistemic and conditional logics. Although this may not be so obvious, first-order calculi with quantifiers are also permitted.

In $\mathcal{L}$, a subset $X$ is fixed to represent the propositions that are in question for the group; this is the agenda, which is the focus of attention in judgment aggregation theory. In all generality, $X$ needs only to be non-empty with at least one contingent formula (not a logical truth or contradiction), and to be closed for negation. The court example, in the discursive dilemma version, involves the agenda

$$
\bar{X}=\{v, b, d, d \leftrightarrow v \wedge b, \neg v, \neg b, \neg d, \neg(d \leftrightarrow v \wedge b)\} .
$$

The theory represents judgments by subsets $B \subset X$ that are initially unrestricted. These judgment sets will be denoted by $A_{i}, A_{i}^{\prime}, \ldots$ for the individuals $i=1, \ldots n$, and $A, A^{\prime}, \ldots$ for the collective. A formula $\varphi$ represents a proposition, in the sense of a semantic object endowed with a truth value, and it also serves to capture the associated judgment, in the sense of a cognitive operation, thanks to the informal translation:

(R) individual $i$ (the collective) makes the judgment $\varphi$ iff $\varphi$ belongs to the judgment set $A_{i}$ (resp. A).

Judgment sets can be subjected to various logical properties. Here we consider only two sets of judgment sets, i.e., the unrestricted set $2^{X}$ and the set $D$ of consistent and complete judgment sets (with completeness being relative to $X$, not $\mathcal{L}$ ). The theory has also investigated the intermediate case of consistent, but not necessarily complete judgment sets. Thus far, it has not been able to relax consistency (see Dietrich and List, 2008).

The last formal concept is the collective judgment function $F$, which associates a collective judgment set to each profile of judgment sets for the $n$ individuals:

$$
A=F\left(A_{1}, \ldots, A_{n}\right) .
$$

We restrict attention to $F: D^{n} \rightarrow 2^{X}$ and $F: D^{n} \rightarrow D$. Taking $D$ to be the range means that the collective sets obey the same stringent logical constraints as the individual ones. This framework is sufficient to capture simple majority voting, as in the court example, and various generalizations. Formally, define formula-wise majority voting as $F_{m a j}: D^{n} \rightarrow 2^{X}$ such that, for every profile $\left(A_{1}, \ldots, A_{n}\right) \in D^{n}$,

$$
\begin{aligned}
& F_{m a j}\left(A_{1}, \ldots, A_{n}\right)=\left\{\varphi \in X:\left|\left\{i: \varphi \in A_{i}\right\}\right| \geq q\right\}, \\
& \text { with } q=\frac{n+1}{2} \text { if } n \text { is odd and } q=\frac{n}{2}+1 \text { if } n \text { is even. }
\end{aligned}
$$


Here, the range is not $D$ because there can be unbroken ties, and so incomplete collective judgment sets, when $n$ is even. More strikingly, for many agendas, the range is not $D$ also when $n$ is odd, because there are inconsistent collective judgment sets, as the court example illustrates. By varying the value of $q$ between 1 and $n$ in the definition, one gets specific quota rules $F_{m a j}^{q}$. One would expect inconsistency to occur with low $q$, and incompleteness with large q. Nehring and Puppe $(2002,2008)$ and Dietrich and List (2007b) investigate these rules in detail.

Having defined and exemplified $F$ functions, we introduce some axiomatic properties they may satisfy.

Systematicity. For all formulas $\varphi, \psi \in X$ and all profiles $\left(A_{1}, \ldots, A_{n}\right)$, $\left(A_{1}^{\prime}, \ldots, A_{n}^{\prime}\right)$, if $\varphi \in A_{i} \Leftrightarrow \psi \in A_{i}^{\prime}$ for every $i=1, \ldots, n$, then

$$
\varphi \in F\left(A_{1}, \ldots, A_{n}\right) \Leftrightarrow \psi \in F\left(A_{1}^{\prime}, \ldots, A_{n}^{\prime}\right) .
$$

Independence. For every formula $\varphi \in X$ and all profiles $\left(A_{1}, \ldots, A_{n}\right)$, $\left(A_{1}^{\prime}, \ldots, A_{n}^{\prime}\right)$, if $\varphi \in A_{i} \Leftrightarrow \varphi \in A_{i}^{\prime}$ for every $i=1, \ldots, n$, then

$$
\varphi \in F\left(A_{1}, \ldots, A_{n}\right) \Leftrightarrow \varphi \in F\left(A_{1}^{\prime}, \ldots, A_{n}^{\prime}\right) .
$$

Monotonicity. For every formula $\varphi \in X$ and all profiles $\left(A_{1}, \ldots, A_{n}\right)$, $\left(A_{1}^{\prime}, \ldots, A_{n}^{\prime}\right)$, if $\varphi \in A_{i} \Rightarrow \varphi \in A_{i}^{\prime}$ for every $i=1, \ldots, n$, with $\varphi \notin A_{j}$ and $\varphi \in A_{j}^{\prime}$ for at least one $j$, then

$$
\varphi \in F\left(A_{1}, \ldots, A_{n}\right) \Rightarrow \varphi \in F\left(A_{1}^{\prime}, \ldots, A_{n}^{\prime}\right) .
$$

Unanimity preservation. For every formula $\varphi \in X$ and every profile $\left(A_{1}, \ldots, A_{n}\right)$, if $\varphi \in A_{i}$ for every $i=1, \ldots, n$, then $\varphi \in F\left(A_{1}, \ldots, A_{n}\right)$.

By definition, $F$ is a dictatorship if there is a $j$ such that, for every profile $\left(A_{1}, \ldots, A_{n}\right)$,

$$
F\left(A_{1}, \ldots, A_{n}\right)=A_{j} .
$$

There can only be one such $j$, to be called the dictator. The last property is

Non-dictatorship. $F$ is not a dictatorship

It is routine to check that $F_{m a j}$ satisfies all the list. Unanimity preservation and Non-dictatorship are self-explanatory. Systematicity means that the group, when faced with a profile of individual judgment sets, gives the same answer concerning a formula $\varphi$ as it would give concerning a possibly different formula $\psi$, when faced with a possibly different profile, supposing that the individual judgments concerning $\varphi$ in the first profile are the same as those concerning $\psi$ in the second profile. Independence amounts to restricting this requirement to $\varphi=\psi$. Thus, it eliminates one claim made by Systematicity - i.e., that the identity of the formula does not matter - while preserving another - i.e., that the 
collective judgment of $\varphi$ depends only on individual judgments of $\varphi$. Monotonicity requires that, when a collective result favours a subgroup's judgment, this still holds if more individuals join the subgroup.

Systematicity, Independence and Monotonicity are clearly reminiscent of neutrality, independence of irrelevant alternatives and positive responsiveness in social choice theory. Each can be defended by normative arguments that have already surfaced there, i.e., systematicity by the fact that many voting rules satisfy it, independence by its non-manipulability property, and monotonicity by its democratic responsiveness implication. The argument for Unanimity preservation follows the existing one for the Pareto principle, while Non-dictatorship is regarded as unpalatable, exactly as in social choice theory.

Systematicity was the condition underlying List and Pettit's (2002) impossibility theorem, but henceforth, the focus of attention shifted to Independence. The problem that has gradually raised to the fore is to characterize - i.e., find necessary and sufficient conditions for - the agendas $X$ such that no $F: D^{n} \rightarrow D$ satisfies Non-dictatorship, Independence, and Unanimity preservation. There is a variation of this problem with Monotonicity as a further condition, and another variation that involves drawing a distinction between premisses and conclusions within the agenda $X$. The next section sketches the respective answers.

\section{The present of the theory: a characterization theorem in three forms}

The promised answers depend on further technical notions. First, a set of formulas $\mathcal{S} \subset \mathcal{L}$ is called minimally inconsistent if it is inconsistent and all its proper subsets are consistent. With the elementary propositional calculus used for the court example, this is the case for

$$
\{v, b, d \leftrightarrow v \wedge b, \neg d\},
$$

but not for

$$
\{\neg v, \neg b, d \leftrightarrow v \wedge b, d\} .
$$

Second, for $\varphi, \psi \in X$, it is said that $\varphi$ conditionally entails $\psi$ - denoted by $\varphi \vdash^{*} \psi-$ if $\varphi \neq \neg \psi$ and there is some minimally inconsistent $Y \subset X$ with $\varphi, \neg \psi \in Y$. This is equivalent to requiring that $\{\varphi\} \cup Y^{\prime} \vdash \psi$ hold for some minimal auxiliary set of premisses $Y^{\prime}$ that is contradictory neither with $\varphi$, nor with $\neg \psi$.

Now, an agenda $X$ is said to be path-connected (or totally blocked) if, for every pair of formulas $\varphi, \psi \in X$, there are formulas $\varphi_{1}, \ldots, \varphi_{k} \in X$ such that

$$
\varphi=\varphi_{1} \vdash^{*} \varphi_{2} \vdash^{*} \ldots \vdash^{*} \varphi_{k}=\psi .
$$


Loosely speaking, agendas with this property have many, possibly roundabout logical connections. Finite agendas can be represented by directed graphs: the formulas $\varphi, \psi$ are the nodes and there is an arrow pointing from $\varphi$ to $\psi$ for each conditional entailment $\varphi \vdash^{*} \psi$. It easy to check that the court agenda $\bar{X}$ is path-connected.

Now, we are in a position to state a first answer to the characterization problem (Dokow and Holzman, 2010a; Nehring and Puppe, 2010a; this result originates in Nehring and Puppe, 2002). From now we assume that $n \geq 2$.

Theorem (first form). If $X$ is path-connected, then no $F: D^{n} \rightarrow D$ satisfies Non-dictatorship, Unanimity preservation, Monotonicity and Independence. The agenda condition is also necessary for this conclusion.

The sufficiency part of this result generalizes the court example in the following sense. The profile of this example shows that, given the agenda $\bar{X}, F_{m a j}$ does not have range $D$. This can be derived abstractly from the sufficiency part by noting that $F_{m a j}$ satisfies the four axiomatic condition and $\bar{X}$ is pathconnected, so that the range condition must fail. The necessity part delivers the significant information that, even keeping $F_{m a j}$, the discursive dilemma cannot occur if $\bar{X}$ is not path-connected.

As it turns out, Monotonicity can be dropped from the list of axioms if the agenda is required to satisfy a further condition. Let us say that $X$ is evennumber negatable if there is a minimally inconsistent set of formulas $Y \subseteq X$ and there are distinct $\varphi, \psi \in Y$ such that $Y_{\neg\{\varphi, \psi\}}$ is consistent, where the set $Y_{\neg\{\varphi, \psi\}}$ is obtained from $Y$ by replacing $\varphi, \psi$ by $\neg \varphi, \neg \psi$ and keeping the other formulas unchanged. This seemingly opaque condition is in fact not very demanding, and for instance $\bar{X}$ satisfies it. To see that, take

$$
Y=\{v, b, d, \neg(d \leftrightarrow v \wedge b)\} \text { and } \varphi=v, \psi=b .
$$

The next result was proved by Dokow and Holzman (2010a). In showing how Arrow's theorem, in one version, can be recovered by judgment aggregation theory, Dietrich and List (2007a) indirectly proved the sufficiency part.

Theorem (second form). If $X$ is path-connected and even-number negatable, then no $F: D^{n} \rightarrow D$ satisfies Non-dictatorship, Unanimity preservation, and Independence. If $n \geq 3$, the agenda conditions are also necessary for this conclusion.

This is often referred to as the "canonical theorem" of judgment aggregation. However, a further step of generalization is available. Unlike the work reviewed so far, it is motivated by the court example in the doctrinal paradox version, as against its discursive dilemma restatement. It is specially devised to clarify the premiss-based method, which is often proposed as a solution to this paradox. 
Formally, we define the set of premisses to be a subset $P \subseteq X$, requiring only that it be non-empty and closed for negation, and we reconsider the framework to account for the difference between $P$ and its complement $X \backslash P$. Adapting the existing conditions, we define

Independence on premisses: same statement as for Independence, but holding only for every $p \in P$.

Non-dictatorship for premisses: there is no $j \in\{1, \ldots, n\}$ such that $F\left(A_{1}, \ldots, A_{n}\right) \cap P=A_{j} \cap P$ for every $\left(A_{1}, \ldots, A_{n}\right) \in D^{n}$.

Similarly revising the agenda conditions, we say that $X$ is path-connected in $P$ if the definition of path-connectedness holds for formulas $\varphi, \psi, \varphi_{1}, \ldots, \varphi_{k}$ that are all in $P$, and say that $X$ is even-number negatable in $P$ if the definition of even-number negatable holds with the negatable pair $\varphi, \psi$ being in $P$. These new conditions can be illustrated in terms of the agenda $\bar{X}$ after fixing a natural interpretation for the set of premisses:

$$
\bar{P}=\{v, b, d \leftrightarrow v \wedge b, \neg v, \neg b, \neg(d \leftrightarrow v \wedge b)\}
$$

As is easy to check, $\bar{X}$ is both path-connected and even-number negatable in $\bar{P}$.

We can now state the last result (Dietrich and Mongin, 2010a).

Theorem (third form). If $X$ is path-connected and even-number negatable in $P$, there is no $F: D^{n} \rightarrow D$ that satisfies Non-dictatorship on premisses, Independence on premisses and Unanimity preservation. If $n \geq 3$, the agenda conditions are also necessary for this conclusion.

This third form of the theorem is more assertive than the second one (take $P=X$, a permitted case). Another relevant specification occurs by taking $X \backslash P$ to be a set of conclusions deducible from $P$ according to the underlying logic. This specification does not make the results any more precise, but it helps connect the theory with those applications - like the doctrinal paradox - in which a logical distinction holds between premisses and conclusions; more on this line below. Having explored our sample theorem in various ways, we now move to the evaluative and prospective part of this chapter.

\section{Law and economics and some future direc- tions for the theory}

Since judgment aggregation theory was motivated by the doctrinal paradox, a law and economics example, it is only natural to investigate its contribution, both current and potential, to that field. As we will show, the current contribution is modest, but various hints suggest a potential. Starting from Kornhauser and Sager's groundbreaking discussion, legal theorists have inquired about the prevalence of the doctrinal paradox in the actual working of U.S. collegiate 
courts, and usually concluded that the paradox, or related forms of conflict between the issue- and case-based methods, occurred rarely. Kornhauser and Sager (1993) found examples in the U.S. Supreme Court record, but striking as they are, they are small in number and even debatable as such (see Post and Salop, 1991-1992, and Nash, 2003, for a critical review). A major defect of these empirical investigations, they do not distinguish sufficiently between the low prevalence of the paradox and the fact that it is difficult to recognize. U.S. collegiate courts do not always state in their final judgments what the judges' individual positions are, and this even holds for the Supreme Court, which is more profuse than any other collegiate court when it comes to making individual positions known.

In view of these uncertainties, an obviously useful preliminary would be to investigate the combinatorics of the doctrinal paradox or related forms of conflict. On the court agenda defined above, granting that the formula $d \longleftrightarrow v \wedge b$ is unanimously agreed, the combinatorics reduces to the simple task of counting how many profiles of judgments on $b, d, v$ give rise to a conflict between the two methods when majority voting applies. It is easy to see that Kornhauser and Sager have exactly recognized which set of profiles precipates the conflict and this set has a relatively low probability ratio (our computation based on standard independence and uniformity assumptions leads to a ratio of $3 / 32$ ). Less trivially, one could let the court agendas vary, and for instance, count how many agendas are path-connected among all those which can be formulated in the propositional language based on the three propositional variables $b, d, v$. More abstract combinatorial investigations could be performed in connection with the theoretical results of last section, and they might deliver useful information for the legal theorists, over and beyond what can be said on the doctrinal paradox itself.

Whatever its mathematical and empirical prevalence, the doctrinal paradox serves the didactic purpose of pointing out two plausible adjudication methods that collective courts can employ, and the law and economics literature has actually spent more time on comparing these methods in general than on the paradox itself. While Kornhauser and Sager (1993) and Kornhauser (2008) express context-sensitive preferences between the two methods, Rogers (1996) supports the case-based method unreservedly, and Post and Salop (1992) support the issue-based method unreservedly. The arguments displayed in this controversy are far from being tight. They tend to entangle formal questions, e.g., which method is more likely to deliver a well-defined solution or more likely to resist strategic manipulation, with substantial legal questions, e.g., whether one method is more likely to protect the defendant in a criminal trial. They also tend to entangle static properties, such as the properties just said, with dynamic properties, such as the ability to define law by precedents, over and beyond the resolution of a particular case. Some writers go as far as to attribute the doctrinal paradox to one of the two methods, whereas the other is obviously co-responsible for it. All in all, despite some analytic efforts like Nash's (2003), 
much remains to be done in order to produce a decent list of normative considerations and weigh them against each other to decide between the two methods. Some formalism would help bring order, but legal theorists do not use any or provide sketches that fall below the mark.

They can be excused by the fact that judgment aggregation theorists have not sufficiently addressed their concerns. We have emphasized from the start that the doctrinal paradox was not the discursive dilemma, and that these theorists have taken the latter, not the former, to be the starting point of their formal investigations. The tendency in their field is to play down the significance of the distinction; this shows in some blurred accounts that motivate the theorems, and in such strange expressions as "the discursive paradox". But legal theorists have little use for the discursive dilemma and its elaboration; what they are really concerned with is the doctrinal paradox and the accompanying comparison of methods to adjudicate legal claims. This explains Dietrich and Mongin's (2010a) unconventional move of distinguishing between premisses and conclusions in their theorem statement. With this logical distinction, they make a step towards formalizing the legal distinction between issues and case. Clearly, the latter presupposes the former, although it goes beyond it semantically. This limited step makes it possible already to draw some lessons from judgment aggregation theory for the legal theorists (see also Mongin, 2008) .

One lesson concerns the highly generalized form of the doctrinal paradox this theorem delivers. It does not mention simple majority voting, which is the only decision rule considered by legal theorists, but abstract conditions that other rules can also obey; in this way, the theorem makes the paradox more acute than the legal theorists have conceived of it. Among these abstract conditions, Unanimity preservation stands apart in the theorem, because it is the only one that applies both to premisses and conclusions; for example, Independence is here restricted to premisses. Heuristically, the source of the impossibility lies in the fact that, when a profile exhibits unanimous endorsement of some conclusion, the collective concurs; this connects the impossibility with the classic problem of "spurious unanimity" in the case of collective preference under uncertainty (Mongin, 1997-2016). The implication for the legal theorists is that judges cannot at the same time apply majority voting on the issues and record their unanimous agreement on the case when this occurs. In other words, the issuebased method already clashes with a very weak form of the case-based method. Since such a limited addition may be difficult to avoid in practice, one may think that the issue-based method is not as safe as it has often been suggested.

Another lesson of interest, which is this time shared by judgment aggregation theory as a whole, is that the agenda plays an essential part in the origin of impossibilities. As the "canonical theorem" and its variants exactly characterize the troublesome agendas, they offer both an explanation of the impossibilities (through the sufficient conditions) and a way of avoiding them (through the necessary conditions). A quick implication for legal theorists is that courts could do 
away with some occurrences of the impossibilities by restructuring their agenda. Experimenting with toy examples suggests that slight changes might be sufficient. However, when they come to practical solutions, legal theorists consider $e x$ post revisions of individual opinions rather than this attractive ex ante solution (see the examples of Supreme Court justices reversing their opinions in Kornhauser and Sager's, 1993, and Post and Salop, 1991-1993). To pursue this point appropriately, careful attention must be payed to the logical status of the legal doctrine (contract law in the court example of the doctrinal paradox). Does it belong to the agenda? If it does, should it also be subjected to a vote if the issue-based method is adopted? And what is the most suitable logic to capture its role in the judges' reasoning? More specifically, is elementary propositional logic sufficient, as in the usual restatement of the court example, or should one rather resort to some more sophisticated logic, say deontic or conditional logic? These are open questions, with some hints of answers in Mongin (2012) following technical advances by Dietrich (2010). Interestingly, some legal theorists - e.g., Landa and Lax (2009) - appear to have foreshadowed some of these apparently abstruse questions. Here is another subarea in which law and economics and judgment aggregation theory could fruitfully join forces.

\section{References}

Dietrich, F. (2006), "Judgment Aggregation: (Im)Possibility Theorems", Journal of Economic Theory 126: 286-298.

Dietrich, F. (2007), "A Generalized Model of Judgment Aggregation", Social Choice and Welfare 28: 529-565.

Dietrich, F. (2010), "The Possibility of Judgment Aggregation on Agendas with Subjunctive Implications", Journal of Economic Theory 145: 603-638.

Dietrich, F. and C. List (2007a), "Arrow's Theorem in Judgment Aggregation", Social Choice and Welfare 29: 19-33.

Dietrich, F. and C. List (2007b), "Judgment Aggregation by Quota Rules: Majority Voting Generalized", Journal of Theoretical Politics 19: 391-424.

Dietrich, F. and C. List (2008), "Judgment Aggregation Without Full Rationality", Social Choice and Welfare 31: 15-39.

Dietrich, F. and P. Mongin (2010a), "The Premiss-Based Approach to Judgment Aggregation", Journal of Economic Theory 145: 562-582.

Dietrich, F. and P. Mongin (2010b), "Un bilan interprétatif de la théorie de l'agrégation logique", Revue d'économie politique, 120, p. 929-972.

Dokow, E. and R. Holzman (2009), "Aggregation of Binary Evaluations for Truth-Functional Agendas", Social Choice and Welfare 32: 221-241.

Dokow, E. and R. Holzman (2010a), "Aggregation of Binary Evaluations", Journal of Economic Theory 145: 495-511.

Dokow, E. and R. Holzman (2010b), "Aggregation of Binary Evaluations with Abstentions", Journal of Economic Theory 145: 544-561. 
Grossi, D. and G. Piggozzi (2014), Judgment Aggregation: A Primer, San Rafael, CA: Morgan \& Claypool.

Guilbaud, G.T. (1952), "Les théories de l'intérêt général et le problème logique de l'agrégation", Économie appliquée, 5, p. 501-584. Reprinted in Revue économique, 63 (2012): 659-720.

Kornhauser, L.A. (2008), "Aggregate Rationality in Adjudication and Legislation", Politics, Philosophy, Economics 7: 5-27.

Kornhauser, L.A. and L.G. Sager (1986), "Unpacking the Court", Yale Law Journal 96: 82-117.

Kornhauser, L.A. and L.G. Sager (1993), "The One and the Many: Adjudication in Collegial Courts", California Law Review 81: 1-59.

Landa, D. and J.R. Lax (2009), "Legal Doctrine on Collegial Courts", Journal of Politics 71: 946-963.

List, C. and P. Pettit (2002), "Aggregating Sets of Judgments: An Impossibility Result", Economics and Philosophy 18: 89-110.

Mongin, P. (1997), "Spurious Unanimity and the Pareto Principle", Thema Working Paper, Université of Cergy-Pontoise (published in Economics and Philosophy 32 (2016): 511-532).

Mongin, P. (2008), "Factoring Out the Impossibility of Logical Aggregation", Journal of Economic Theory 141: 100-113.

Mongin, P. (2012), "The Doctrinal Paradox, the Discursive Dilemma, and Logical Aggregation Theory", Theory and Decision, 73: 315-345.

Nash, J.R. (2003), "A Context-Sensitive Voting Protocol Paradigm for Multimembers Courts", Stanford Law Review 56: 75-159.

Nehring, K. and C. Puppe (2002), "Strategy-Proof Social Choice on SinglePeaked Domains: Possibility, Impossibility and the Space Between", WP of the Dept of Economics, University of California, Davies.

Nehring, K. and C. Puppe (2008), "Consistent Judgement Aggregation: The Truth-Functional Case", Social Choice and Welfare 31: 41-57.

Nehring, K. and C. Puppe (2010), "Abstract Arrowian Aggregation", Journal of Economic Theory 145: 467-494.

Pauly, M. and M. van Hees (2006), "Logical Constraints on Judgment Aggregation", Journal of Philosophical Logic 35: 569-585.

Pettit, P. (2001), "Deliberative Democracy and the Discursive Dilemma", Philosophical Issues 11: 268-299.

Pigozzi, G. (2017), "Belief Merging and Judgment Aggregation", The Stanford Encyclopedia of Philosophy (Winter 2016 Edition), E.N. Zalta (ed.),

$<$ https://plato.stanford.edu/archives/win2016/entries/belief-merging/>.

Post, D. and S.C. Salop (1991-1992), "Rowing Against the Tidewater. A Theory of Voting by Multijudge Panels", Georgetown Law Journal: 80, 743774 .

Rogers, J.M. (1996), ""Issue-Voting" by Multi-member Appellate CourtsA Response to Some Radical Proposals", Vanderbilt Law Review: 49 . 997-1044.

Rubinstein, A. and P.J Fishburn (1986), "Algebraic Aggregation Theory", Journal of Economic Theory: 38, 63-77. 